\title{
On Asymptotic Elias Bound for Euclidean Space Codes over Distance-Uniform Signal Sets
}

\author{
G. Viswanath \\ Dept. of Electrical Comm. Engg. \\ Indian Institute of Science \\ Bangalore, 560 012, India \\ gviswa@protocol.ece.iisc.ernet.in
}

\author{
B. Sundar Rajan \\ Dept. of Electrical Comm. Engg. \\ Indian Institute of Science \\ Bangalore, 560 012, India \\ bsrajanqece.iisc.ernet.in
}

\begin{abstract}
The asymptotic Elias upper bound of codes designed for Hamming distance is well known. Piret [3] and Ericsson [4] have extended this bound for codes over symmetric $P S K$ signal sets with Euclidean distance and for codes over signal sets that form a group, with a general distance function respectively. The tightness of these bounds depend on a choice of a probability distribution, and finding the distribution (called optimum distribution henceforth) that leads to the tightest bound is difficult in general. In [1] these bounds were extended for codes over the wider class of distance-uniform signal sets. In this paper we obtain optimum distributions for codes over signal sets matched [2] to (i) dihedral group, (ii) dicyclic group (iii) binary tetrahedral group (iv) binary octahedral group (v) binary icosahedral group and (vi) n-dimensional cube. Further we compare the bounds of codes over these signal sets based on the spectral rate.
\end{abstract}

\section{INTRODUCTION}

A signal set is said to be distance-uniform if the Euclidean distance distribution of all the points in the signal set from a particular point in the signal set is same from any point. The class of signal sets matched to groups form an important class of distance-uniform signal sets. The existence of distanceuniform signal sets that are not matched to any group was shown by Slepian [5]. In [1] it is shown that the bounds of Piret[3] and Ericsson[4] hold for the wider class of distanceuniform signal sets. These bounds and their tightness depends on the choice of a probability distribution.

Theorem 1: [1] Let $A$ be a distance-uniform signal set with $M$ signal points $\left\{a_{0}, a_{1}, \ldots, a_{M-1}\right\}$ and $S$ be a $M \times M$ matrix with $(i, j)^{\text {th }}$ entry $s_{i j}$ equal to $d_{i, j}^{2}$, the squared Euclidean distance between $a_{i}$ and $a_{j}$. For $C$, a length $n$ code over $A$, let

$$
\begin{array}{r}
\delta(C)=\frac{1}{n} d^{2}(C), \quad R(C)=\frac{1}{n} \ln |C| \text { and } \\
R(M, \delta)=\lim _{n \rightarrow \infty} \sup _{\substack{|C| \geq n \\
\delta(C) \geq \delta}} R(C)
\end{array}
$$

$d^{2}(C)$ is the minimum squared Euclidean distance (MSED) of the code. The asymptotic upper bound $R_{U}(M, \delta)$ on $R(C)$ is given in terms of a probability distribution $\underline{\beta}=$ $\left(\beta_{0}, \beta_{1}, \cdots, \beta_{M-1}\right)$, by

$$
R_{U}(M, \delta)=\ln (M)-H(\underline{\beta}) \text { and } \delta=\underline{\beta} S \underline{\beta}^{T}
$$

where $H(\underline{\beta})=-\Sigma_{i=0}^{M-1} \beta_{i} \ln \left(\beta_{i}\right)$

${ }^{1}$ This work was partly supported by CSIR, India, through Research Grant (22(0298)/99/EMR-II) to B.S.Rajan.

\section{Optimum Distribution for certain Distance UNIFORM Signal SETS}

In [1] the optimum distributions for codes over simplex signal sets and biorthogonal signal sets were obtained. It turns out that the optimum distribution for codes over signal sets matched to cyclic group, dihedral group, dicyclic group, binary tetrahedral group and Slepian signal sets in five and six dimensions [5] is similar to those obtained for simplex and biorthogonal signal sets in [1]. The optimum distribution is

$$
\beta_{r}(\mu)=\frac{e^{-\mu d^{2}(r)}}{\sum_{s=0}^{M-1} e^{-\mu d^{2}(s)}} r=0,1,2, \cdots, M-1
$$

where $d^{2}(r)$ is the squared Euclidean distance between the 0 th point and the $r$ th point of the $M$ point signal set. The positive parameter $\mu$ determines the value of $\delta$ for which the distribution is optimum by $\delta=\sum_{r=0}^{M-1} \beta_{r}(\mu) d^{2}(r)$.

\section{Discussions}

For band-limited applications, the rate of the code per dimension is the appropriate parameter based on which signal sets are to be compared. To facilitate this we measure the rate of the code per two dimensions, i.e.

$$
\frac{2}{N} R(C)=\frac{2}{N n} \ln |C|
$$

and call it the spectral rate (rate in bits per symbol per two dimensions) of the code. In terms of this spectral rate it turns out that codes over signal sets matched to binary tetrahedral, binary octahedral and binary icosahedral group have better bounds compared to codes over signal sets matched to dicyclic and dihedral groups and $n$-dimensional cube.

\section{REFERENCES}

[1] B. Sundar Rajan and G. Viswanath, 'Asymptotic Elias Bound for Euclidean Space Codes over Distance Uniform Signal Sets', accepted for publication in IEICE Trans. on Fundamentals of Information, Communication and Computing

[2] H.A. Loeliger, ' Signal Sets Matched to Groups', IEEE Trans. Information Theory, Vol.IT-37, No.6, Nov.1991, pp.1675-1682.

[3] P. Piret, ' Bounds for Codes Over the Unit Circle', IEEE Trans. Information Theory, Vol.IT-32, No.6, Nov.1986, pp.760-767.

[4] T. Ericsson, 'Generalizations of the Johnson and the BassalygoElias bounds', Fourth Joint Swedish-Soviet International Workshop on Information Theory', Aug. 27- Sept. 1, 1989, Gotland, Sweden.

[5] D. Slepian, 'On neighbor distances and symmetries in group codes', IEEE Trans. Inform. Theory, Vol.17, pp.630-632, Sept. 1971. 